\title{
Hybrid Segmentation and 3D Modeling of Pleural Effusion on CT
}

\author{
${ }^{1}$ K.P.Ashvitha, ${ }^{2}$ M.ShilpaAarthi, ${ }^{3}$ M.R.Thamizhkkanal, ${ }^{4}$ M.Rajendiran, ${ }^{5}$ S.Malathi \\ ${ }_{1,2,3} \mathrm{PG}$ scholar, ${ }^{4,5}$ Professor \\ ashvithachitty@gmail.com,shilpaaarthidaisy@gmail.com,charuvarshini995@gmail.com, \\ muthusamyrajendiran@gmail.com,malathi_raghu@hotmail.com \\ Dept of CSE, Panimalar engineering college, Chennai
}

\begin{abstract}
Pleural effusion is the excess fluid within the pleural space. Pleural effusion detection helps in the diagnosis of diseases. If the effluent is not within the safe zone then it creates many problems including the death of human. The pleural level can be detected manually which is time consuming. The objective of this study is to determine the pleural fluid on computed tomography (CT) scan images automatically. The pleural space is segmented by parietal pleura extraction and visceral pleura extraction. The method is based on nonlinear anisotropic diffusion filtering and hybrid segmentation. 3D deformable modeling is applied for three dimensional view of the pleural effusion. We compare this method to manual segmentations and result is closer as expected. This method is useful in diagnosis of pleural effusion effectively rather than manual determination.
\end{abstract}

Index Terms-pleural effusion, nonlinear anisotropic diffusion, segmentation

\section{INTRODUCTION}

$\mathrm{P}$ leural Effusion (PE) is an abnormal measure of fluid around the lung. Pleural radiations can realize various restorative conditions. The pleura is a thin film that lines the surface of the lungs and within the chest divider outside the lungs. In pleural radiations, liquid displays in the space between the layers of pleura. By and large, just teaspoons of liquid are available in the pleural space which enables the lungs to move easily inside the chest depression amid relaxing.The parietal pleura keep running along within the chest divider and the instinctive pleura cover the lungs. Both the size and area of the emanation can be symptomatically huge and imperative to understanding consideration. In crisis circumstances, radiologists are solicited to evaluate the volume from pleural liquid accumulations on CT. The extent of a radiation can fundamentally affect persistent reports, particularly in basically sick patients. For CT, division is viewed as standard strategy to measure liquid accumulations, both in the chest and the Abdomen. The strategies to evaluate pleural radiations on CT ought to be sufficiently straightforward to be recollected, simple to perform, and adequately exact to help clinicians in their choices in regards to quiet reports.

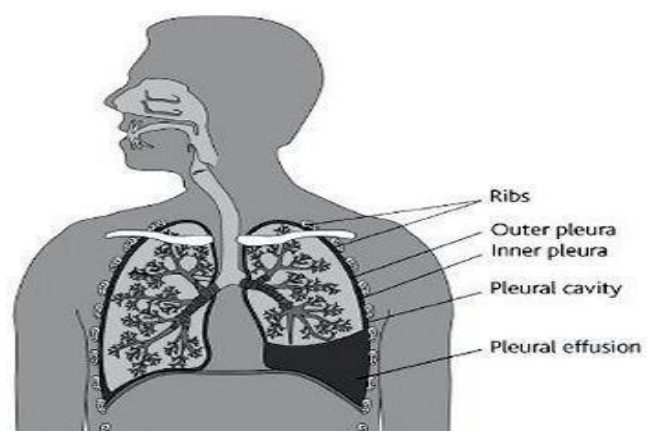

Fig 1: Depicts chest with Pleural Effusion

\section{RELATED WORKS}

At first Chest $\mathrm{x}$-beams are the most generally utilized examination to evaluate for nearness of a pleural emanation, however it ought to be noticed that on a routine erect chest $\mathrm{x}$ beam as much as $250-600 \mathrm{ml}$ of liquid is required before it winds up clear. Later on ultrasound permits the discovery of little measures of pleural emission liquid with positive ID of sums as little as 3 to $5 \mathrm{ml}$ that can't be distinguished by $\mathrm{x}$ beams, which is equipped for identifying volumes over $50 \mathrm{ml}$ of fluid. Contrasting with the radiological technique, ultrasound permits a basic separation of pleural emission fluid and thickened pleura.

Figured tomography (CT) is phenomenal at recognizing little measures of liquid and is additionally regularly ready to distinguish the measure of liquid present with the assistance of CT check picture. While comparing to chest X-rays, CT scan is more efficient and effective. Subsequently clinically more nitty gritty data about pleural emanations and numerous other lung variations from the norm can be resolved. In the greater part of the cases, the exactness of CT settles on it the better decision.This technique gives an approach to decide the volume of pleural radiation by fragmenting the chest CT filter pictures. 
Several papers have been proposed on pleural effusion analysis with automatic segmentation. Donohue et al. proposed cut by-cut obliged locale based division, and can likewise fragment aspiratory masses. Qian et al. proposed cubic Hermite bend fitting and bouncing box calculation, utilizing the focal segment of the picture, and a Bessel technique estimation of limit focuses. Kido and Tsunomori utilized a restrictive district developing calculation.John Bliton, Jianhua Yao proposed a paper on pleural effusion detection with automatic segmentation. They followed the process of segmenting the chest CT scan images and then validated the results with manual segmentationThe pleural space is fragmented on every 2-D cut by recognizing the instinctive and parietal pleura. The instinctive pleura are identified utilizing the lung as point of interest, and a dynamic form display (ACM) is utilized to refine the recognition.But their method is complicated with advancement in image processing. Thresholding and region grow segmentation will now work with their proposal. In order to that they followed Bernstein polynomial curve for pleura segmentation. Dynamic shape show is likewise used to refine instinctive layer amid division.

\section{PROPOSED WORKS}

The detection of pleural effusion is done by segmenting the chest CT scan. Previous studies used custom developed software for segmentation but we use hybrid segmentation method for effective determination of pleural effusion. A slice of chest CT scan image is first preprocessed to reduce noise. Later on refined image is used to segment the visceral and parietal pleura. This bend is then disfigured in the route expected to diminish the cost along these lines pushing the bend toward a position where fetched is privately limited. Presumably this happens when the bend is accurately arranged on the question. Now the segmented layer is used for validation. A set of segmented slices are formatted into 3D representation.

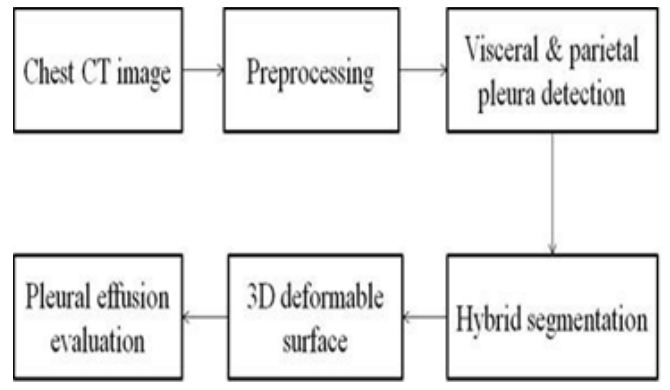

Fig 2: Systemic flow of pleural effusion determination

\section{A) PREPRoCEssing}

At first the CT examine picture contains clamor, they influence the division result while utilizing dim based level division methods. So as to enhance the division the commotion ought to be diminished.Nonlinear anisotropic diffusion filtering is used so that the image intensity could be smoothed by diffusion. Other filters determine the gradient based on some local features, but here the diffusion should preserve edges. In Nonlinear anisotropic dissemination lean towards parallel dispersion to edges. One of the highlights is nonlinearity where the dispersion at fringe is considerably less than the dissemination somewhere else. Other is anisotropy where dissemination ought to be opposite to the edge. We want to stop the diffusion over the edge. Therewith we need as one eigenvector the direction of the gradient. The second is perpendicular and it can be seen as the tangential vector to the edge.

$$
\begin{gathered}
v 1=\frac{\nabla u}{|\nabla u|} \\
v 2=\left(\begin{array}{c}
{[v 1] y} \\
-[v 1] x
\end{array}\right)
\end{gathered}
$$

Where $v 1 \| \nabla \mathrm{u}$

$$
v 2 \perp \nabla u
$$

$$
\alpha 1=g\left(|\nabla u|^{2}\right) \text { and }
$$

$\propto 2=1$ Now we obtain D as

$$
D=\left(\begin{array}{cc}
\mid & \mid \\
v 1 & v 2 \\
\mid & \mid
\end{array}\right) \cdot\left(\begin{array}{cc}
\propto 1 & 0 \\
0 & \propto 2
\end{array}\right)\left(\begin{array}{cc}
\mid & \mid \\
v 1 & v 2 \\
\mid & \mid
\end{array}\right)
$$

Hence the result is an edge enhancing nonlinear anisotropic diffusion and the original image has been retained.

\section{B) LUNG SEGMENTATION}

The pleural emission is available between the instinctive and pleural layers of the pleura. The parietal pleura (external layer) are connected to the chest divider and the instinctive pleura (inward layer) cover the lung. Segmentation is done in three steps. Initially the visceral layer is detected and second is to detect parietal pleura layer. Final step is to segment the pleura, which is done by Hybrid segmentation. The method begins by obtaining closed unit-width segment boundaries using an optimum edge detection method suitably modified to detect the edges in the pleura space. The key suspicion that we make about articles to be divided by this system is this: At each point on the genuine edge of a protest, adjacent focuses inside and outside the question will be displayed well by the mean powers of the neighborhood districts. The outcome is a vitality that is more worldwide in nature than edgebased streams. The cost of the whole bend is regularly 
called 'vitality'. In the meaning of this half and half vitality we utilize a few documentations. ' $x$ ' and 's' speak to free spatial factors and I speak to the picture. Omega and Omega-bar speak to the inside and outside of the bend individually.

The two bends (instinctive and parietal layers) with endpoints along these lines created are associated with straight lines to frame a shut shape and all pixels that fall inside are characterized as PE. Once the pleural space is portioned on every 2-D cut, the edges of the whole PE division are equally tested. These are utilized as control focuses to produce a Bezier surface.

$$
p(u, v)=\sum_{i=0}^{n} \sum_{j=0}^{m} Z_{i}^{n}(u) Z_{j}^{m}(v) K_{i, j}
$$

where $\mathrm{p}(\mathrm{u}, \mathrm{v})$ is a point at first glance, $(\mathrm{n}, \mathrm{m})$ is the level of the surface, which differs with the quantity of control focuses, $\mathrm{Z}$ is the Bezier surface capacity, and $\mathrm{ki}, \mathrm{j}$ is the arrangement of control focuses. This procedure smoothes the division and adjusts certain blunders in the underlying division.

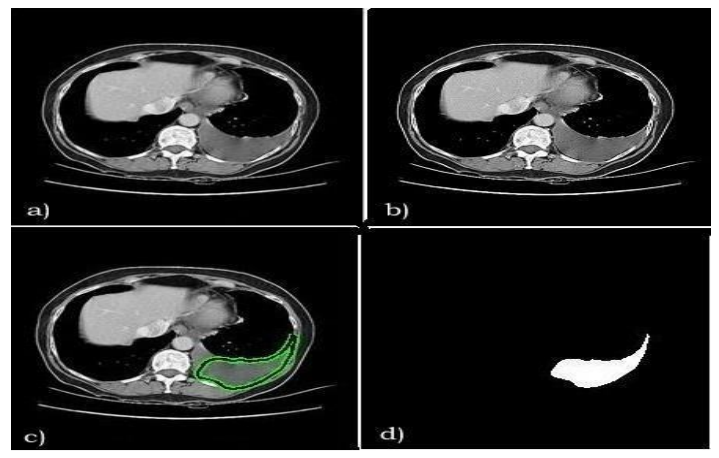

Fig 3: a) Original image. b) After preprocessing. c) Pleura segmentation. d) Resulting PE segmentation

\section{C) 3D DEFORMABLE SURFACE}

A set of $2 \mathrm{D}$ slices are segmented, now the segmented pleural surface are to form a deformable 3D surface. A stack of $2 \mathrm{D}$ images of dimension a $\mathrm{x} b$ each, a simple loop can be used to create a 3D array. It contains planar curves and it ensures smooth and coherent surface between the slices. The array $\mathrm{X}, \mathrm{Y}$, and $\mathrm{Z}$ defines the coordinates for the volume $\mathrm{V}$. The structure contains the faces and vertices of the isosurface, which can be passed directly to the slices to form 3D representation.With the simplified 3-D model, the curve on one slice is also attracted by the edge data of the neighboring slices and this helps to reconstruct the whole curve. This 3D model shows the variation in manual segmentation volume and automatic segmentation volume by this proposed method.

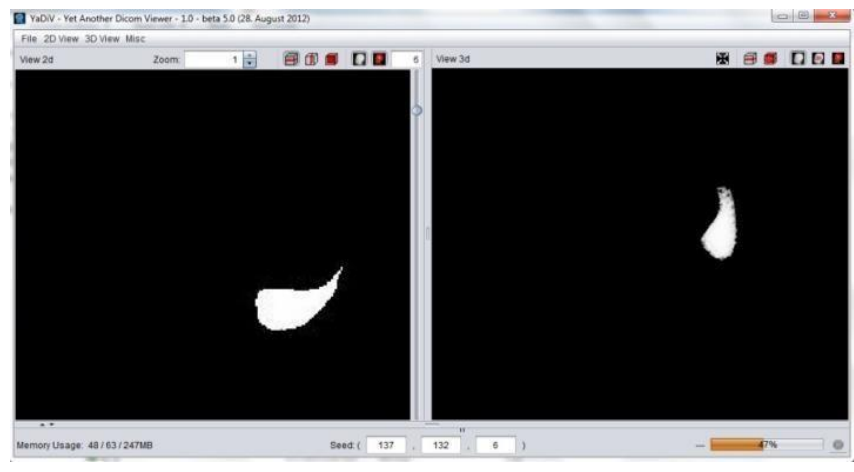

Fig 4: 3D Deformable surface using YaDiv

\section{RESULT AND ANALYSIS}

A strategy to portion and measure the volume of pleural emission utilizing chest CT picture is proposed. In this nonlinear anisotropic dispersion channel is utilized as a part of the pre-preparing stage. With the goal that the clamor introduce in unique picture will be decreased to a degree.This preprocessed image is used to segment area more accurately with its edges. This method is very effective and efficient than anisotropic diffusion filter. It shows better behavior near the edges.

The hybrid segmentation method determines the edges of the pleural space with its relating sequence of area. But in the existing stage, Bezier surface fitting algorithm was used. It extracts the parietal pleura and visceral pleura for pleural space segmentation.

The segmented slices are now evaluated with manual segmentation. For each cut total volume on modified division, mean volume on manual division, volume got in manual division bestowed to customized division and total cover volume.

$$
\begin{aligned}
& \text { Dice }=2 * \text { OLV } /(\text { TMV }+ \text { TAV }) \\
& \text { Cover }=\text { OLV } / T M V
\end{aligned}
$$

Dice coefficients, percent overlap, total manual volume (TMV) and total automation volume (TAV) are obtained from above equation with corresponding slices. Ordinary pleural liquid has the reasonable ultra filtrate of plasma that begins from the parietal pleura. In our approach CT scanning is superior to plain radiographs in the segmentation of pleura space. It is particularly helpful in the assessment and management of located pleural effusions. Hence the segmented pleura space will have shape with smooth margins and relatively homogeneous attenuation. 
TABLE 1

Comparison of Dice coefficient

\begin{tabular}{|c|c|c|c|}
\hline Dice & $\begin{array}{c}\text { Hybrid } \\
\text { Segmentation }\end{array}$ & $\begin{array}{c}\text { Region } \\
\text { growing }\end{array}$ & OI \\
\hline $\begin{array}{c}\text { Hybrid } \\
\text { Segmentation }\end{array}$ & $\mathrm{X}$ & 0.84 & 0.80 \\
\hline $\begin{array}{c}\text { Region growing } \\
\text { ROI }\end{array}$ & 0.75 & $\mathrm{x}$ & 0.70 \\
\hline & 0.66 & 0.62 & $\mathrm{x}$ \\
\hline
\end{tabular}

Table 1 represents the comparison of dice coefficient with region growing, ROI and hybrid segmentation. The enhanced program had the nearest match to add up to division volume. Additionally, there was less fluctuation in all out volume for the correlations with the programmed strategies than among the manual divisions. In our approach CT scanning is superior to plain radiographs in the segmentation of pleura space. It is particularly helpful in the assessment and management of located pleural effusions.

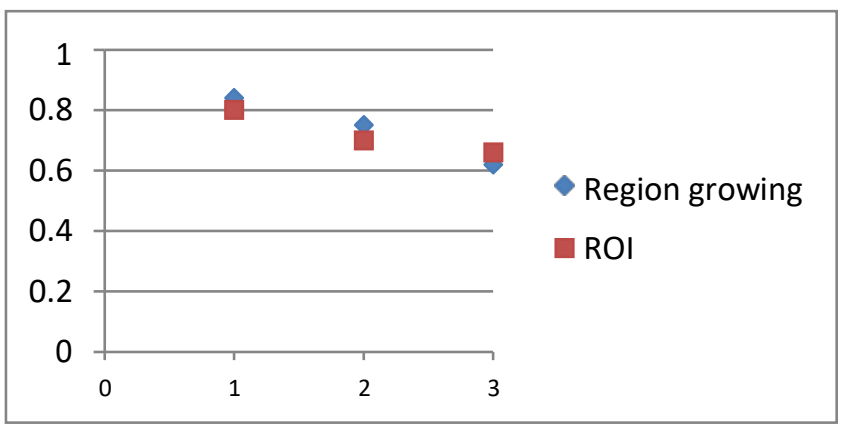

Fig 5: Comparison of dice coefficient with Hybrid Segmentation

Figure 5 plots the dice coefficient comparison of hybrid segmentation with region growing and ROI methods. There is slight variation between hybrid segmentation and other methods. The pleural effusion volume determined by manual and hybrid segmentation in each CT scan is represented in Table 2.
TABLE 2

Comparison of Pleural volume $(\mathrm{mL})$

\begin{tabular}{|c|c|}
\hline $\begin{array}{c}\text { Hybrid } \\
\text { Segmentation }\end{array}$ & Manual \\
\hline 132 & 100 \\
\hline 148.5 & 130 \\
\hline 165 & 150 \\
\hline 205 & 200 \\
\hline
\end{tabular}

Table 2 depicts the comparison of pleural volume with hybrid segmentation and manual determination. Based on the segmented pixels, the pleural volume is determined. Hence the results are varying from manual determination.

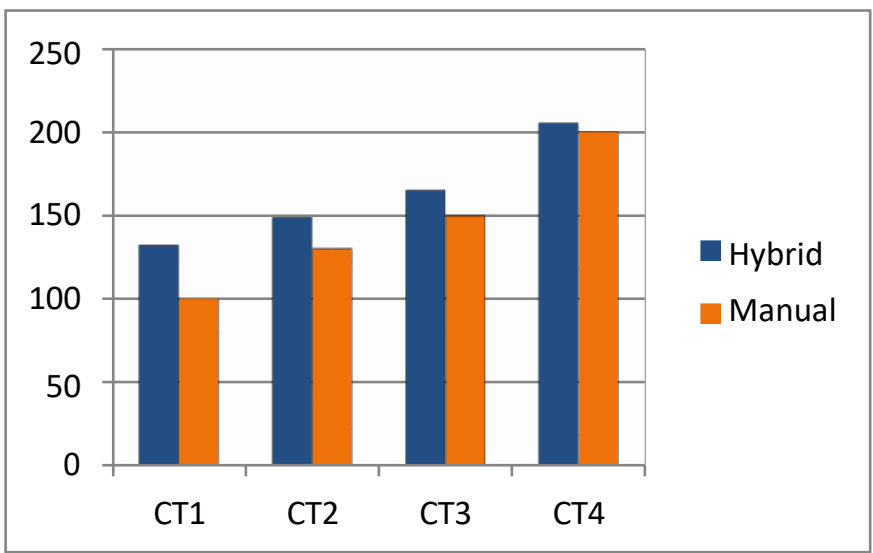

Fig 6: Comparison of Pleural volume

Figure 6.2 depicts the comparison of pleural volume with hybrid segmentation and manual segmentation. It clearly shows the difference in pleural volume. The arrangement of fragmented cuts are utilized to shape a three dimensional perspective of the pleural space, which give better portrayal of the span of the pleural emanation. With the goal that the pleural emission introduced in the lung can be disfigured outwardly than the 2D picture. As opposed to addressing just in the 2D organize, 3D see has more information about the size and condition of the pleural spread. The outcomes demonstrate that the proposed technique to section and measure the volume of pleural emanation is more successful and proficient than existing strategy. 


\section{CONCLUSION}

Our proposed approach in detection pleural radiation is equipped for searching locally for adjust arrangements while comparing with manual segmentations. The described method has been obtained by combining gradient-based and regionbased methods. It makes the segmentation results robust, since the segmentation is done automatically.

The strategy displayed here is just the main utilization of another class of pleural effusion around hybrid segmentation and 3D deformable surface. The methodologies for pleural emanation division talked about in this survey can be positioned based on relevance, precision, execution. The program works best with pleural outflows that have a specific shape, it starts on the posterior of the lung, before its inferior perspective, and they extend diving without moving in the front bearing underneath the base of the lung.The past adaptations incorporate locale based developing and it provides division on limit esteems happened by the commotion. To conquer this issue, direct anisotropic dispersion is utilized and after that the procedure is been finished with crossover division. Our proposed demonstrate needs not so much time but rather more precise than manual division which is done clinically.

\section{REFERENCES}

[1] J. Yao, et al., "Computer-Aided evaluation of Pleural Effusion Using Chest CT Images," IEEE Int. Symposium on Biomedical Imaging, Boston, MA, pp. 241-244, 2009.

[2] Yao J, Bliton J, and Ronald M. Automatic segmentation and measurement of pleural effusions on CT ,in Proc. IEEE Transactions on Biomedical Engineering, 2013; 60(7).

[3] Y. Qian, C. Zhang, and X. Yang, "New method for quantification of pleural effusions from CT imaging," in Proc. ISECS Int. Colloq. Comput. Commun. Control Manage, 2008, pp. 768-773.

[4] Weinheimer O, Achenbach T,et.al, Automatic Lung Segmentation in MDCT Images", Springer-Verlag and Springer,London,2009.

[5] N. Otsu, "A Threshold selecting method from gray- level histograms," IEEE Transactions on Systems, Man, and Cybernetics, vol. 9, no. 1, pp. 62-66, 1979.

[6] D. Jayadevappa, S. Srinivas Kumar, and D. S. Murthy, "A Hybrid segmentation model based on watershed and gradient vector flow for the detection of Brain tumor," International Journal of Signal Processing, Image Processing and Pattern Recognition, vol. 2, no. 3, pp. 29-42, 2009.

[7] H. S. Prasantha, H. L. Shashidhara, K. N. B. Murthy, and L. G. Madhavi, "Medical image segmentation,"International Journal on Computer Science and Engineering, vol. 2, no. 4, pp. 1209- 1218, 2010.

[8] von Falck, S. Meier, S. Jordens, B. King, M. Galanski, and H. O. Shin, "Semiautomated segmentation of pleural effusions in MDCT datasets," Acad. Radiol., vol. 17, pp. 841-848, 2010.

[9] R. Donohue, A. Shearer, and J. Bruzzi, "Constrained region-based segmentation of pleural effusion in thin- slice CT," in Proc. IEEE $13^{\text {th }}$ Int.Mach. Vis. Image Process. Conf., 2009, pp. 24-29.

[10] J.Ivins and J.Porrill ," Active region segmenting medical images"Artifical intelligence vision research unit, vol.94.1994
[11] S. T. Acton, "Multigrid anisotropic diffusion," IEEE Trans. ImageProcess., vol. 7, no. 3, pp. 280-291, Mar. 1998

[12] J. Weickert, Anistropic Diffusion in image processing .Stuttgart,Germany: B.G Teubner,1998

[13] S. Kido and A. Tsunomori, "Automated extractions of pleural effusion in three dimensional thoracic CT images," SPIE Med. Imaging, vol. 7260, pp. 72600D1-8, 2009

[14] B. Li and S.T. Acton, "Active contour external force using vector field convolution for image segmentation," IEEE Trans. Image Process., vol. 16, no. 8, pp. 2096-2106, Aug. 2007.

[15] J. A. Ruskin, J. W. Gurney, M. K. Thorsen, and L. R. Goodman, "Detection of pleural effusions on supine chest radiographs," Amer. J. Roentgenol., vol. 148, pp. 681-683, 1987.

[16] J. Porcel and M. Vives, "Etiology and pleural fluid characteristics of large and massive effusions," Chest, vol. 124, pp. 978-983, 2003.

[17] Sullivan, G D; Worrall. A D; Hockney, R W: Baker. K D: 1990. Active Contours In Medical Image Processing Using A Networked SIMD Array Processor. First British Machine Vision Conference: pp 395-400.

[18] L. Alvarez, P. L. Lions, and J. M. Morel, "Image selective smoothing and edge detection by nonlinear diffusion," SIAM J. Num. Anal., vol. 29, pp. 845-866, 1992

[19] V. Caselles, F. Catte, T. Coll, and F. Dibos, "A geometric model foractive contours," Numer. Math., vol. 66, pp. 1-31, 1993.

[20] L.W.Hedlund, R.F.Anderson, P.L.Goulding, J.W.Beck, E.L.Effmann and C.E.Putman "Two methods for isolating the lung area of a CT scan for density information", Radiology,vol.144 ,pp. 353-357,1982 